\title{
Efficacy of anti-PD-1/PD-L1 antibodies after discontinuation due to adverse events in non-small cell lung cancer patients (HANSHIN 0316)
}

Motoko Tachihara ${ }^{1 *}$, Shunichi Negoro ${ }^{2}$, Takako Inoue ${ }^{3}$, Motohiro Tamiya $^{3}$, Yuki Akazawa ${ }^{4}$, Takeshi Uenami ${ }^{4}$ Yoshiko Urata ${ }^{5}$, Yoshihiro Hattori ${ }^{5}$, Akito Hata ${ }^{6}$, Nobuyuki Katakami ${ }^{6}$ and Soichiro Yokota ${ }^{4}$

\begin{abstract}
Background: Immune checkpoint inhibitors (ICls) have emerged as promising therapeutic agents in non-small cell lung cancer (NSCLC). However, the duration for which ICls should be continued remains a clinical problem.

Methods: We examined the efficacy of anti-PD-1/PD-L1 inhibitors after the discontinuation of antibodies due to adverse events (AES) in patients with NSCLC. This was a multicenter retrospective study that analyzed NSCLC patients who were treated with PD-1/PD-L1 inhibitors by August 2016.

Results: The patients with NSCLC were 18 males and 1 female at a median 67 years of age (range: 49-80 years). Eighteen of 19 patients were treated with nivolumab, one was with atezolizumab. Approximately half of AEs were interstitial pneumonia. Fourteen patients (73.7\%) were treated with steroid therapy. The median number of treatment cycles was 7 (range, 1-70), and the median duration of treatment was 2.8 months (range, 1 day-32. 9 months). The overall response rate with confirmation during treatment was $21.1 \%$. The median progressionfree survival (PFS) was 10.2 months (95\% confidence interval $[\mathrm{Cl}]=3.2-17.1$ months) and 5.6 months $(95 \% \mathrm{Cl}=$ 0-12.2 months) from the initiation and the discontinuation of PD-1/PD-L1 treatment, respectively. The median PFS after discontinuation according to the confirmed response during administration was not reached for partial response (PR) and 4.9 months $(95 \% \mathrm{Cl}, 3.7-6.0)$ for stable disease (SD) patients $(P=0.02)$.
\end{abstract}

Conclusion: The PFS of the PR patients was completely different from that of the SD patients. The cases with PR prior to the onset of AE tended to show a durable response after the discontinuation of PD-1/PD-L1 inhibitors.

Keywords: Anti-PD-1/L1 inhibitor, Non-small cell lung cancer, Adverse event, Discontinuation

\section{Background}

Recent progress in the treatment of advanced nonsmall cell lung cancer (NSCLC) has been remarkable and promising. The development of immunotherapy resulted in a paradigm shift for the treatment of NSCLC. The PD-1 receptor is an immune checkpoint inhibitor expressed on activated $\mathrm{T}$ cells that downregulates excessive immune responses $[1,2]$. Binding of PD-1 to its ligands on tumor cells suppresses $\mathrm{T}$ cells through

\footnotetext{
* Correspondence: mt0318@med.kobe-u.ac.jp

${ }^{1}$ Department of Respiratory Medicine, Kobe University Graduate School of Medicine, 7-5-1, Kusunoki-Cho, Chuo-Ku, Kobe-city, Hyogo 650-0017, Japan Full list of author information is available at the end of the article
}

negative feedback, leading to escape from the immune response [3-5]. Immune checkpoint inhibitors (ICIs), including anti-PD-1/PD-L1 antibodies, aim to restore antitumor immunity and have shown good clinical responses and an improved overall survival (OS) in patients with several tumors, such as melanoma and lung cancer [6-12]. In addition, ICIs notably show a durable clinical benefit persisting long after the cessation of therapy in several tumors [10-16].

Treatment with anti-PD-1/PD-L1 antibodies is associated with toxicities known as immune-related adverse events (irAEs) [6-17]. The typical median time from therapy initiation to irAEs was reported to be within 3

(c) The Author(s). 2018 Open Access This article is distributed under the terms of the Creative Commons Attribution 4.0 International License (http://creativecommons.org/licenses/by/4.0/), which permits unrestricted use, distribution, and 
months, but the frequency of AEs increase with continued therapy $[8,13]$. Thus, it is important to determine the appropriate period of administration of anti-PD-1/ PD-L1 antibodies.

To estimate this period, we retrospectively evaluated the efficacy of PD-1/PD-L1 inhibitors after the discontinuation of these antibodies due to AEs in patients with NSCLC at Japanese cancer research institutes.

\section{Methods}

\section{Patients and treatment}

Patients with advanced NSCLC who received either single-agent anti-PD-1 or PD-L1 antibody until August 31, 2016, and stopped due to AEs at institutions participating in the Hanshin Cancer Research Group were included. Anti-PD-1 antibody, nivolumab was administered at $3 \mathrm{mg} /$ kg every 2 weeks and anti-PD-L1 antibody, atezolizumab was at $1200 \mathrm{mg} /$ body every 3 weeks. Patients' medical records were retrospectively reviewed, and the following information was retrieved: age, gender, lung cancer histology, Eastern Cooperative Oncology Group (ECOG) performance status (PS), smoking status, treatment line, treatment for AEs, and number of treatment cycles. The histological classification of lung cancer was defined based on the World Health Organization pathology classification. Routine chest radiography was conducted every cycle to evaluate the treatment responses and AEs.

\section{Clinical analyses}

Chest computed tomography (CT) was performed every 4 to 16 weeks as a routine procedure and were used to confirm disease response or progression. The treatment effect was evaluated with the Response Evaluation Criteria in Solid Tumors, version 1.1 (RECIST 1.1). Clinical response to therapy, the progression-free survival (PFS), and the OS were evaluated. This study was approved by the ethics committee of Kobe University and each institution.

\section{Statistical analyses}

To analyze the OS and PFS, survival curves were plotted using the Kaplan-Meier method. The PFS with antiPD-1/PD-L1 antibodies was assessed from the initiation of PD-1/PD-L1 treatment to the day on which the first objective signs of disease progression or death were recorded. The OS was calculated from the date of initiation of the anti-PD-1/PD-L1 treatment to the date of death and was censored at the date of last visit for patients whose deaths could not be confirmed. The survival probabilities between groups were compared using a log-rank test. All analyses were run PASW statistics version 18. A spider plot depicted the change from baseline for tumors for each subject in a study by week.

\section{Results}

Patients characteristics

Of the 192 patients who underwent anti-PD-1/PD-L1 treatment, we analyzed 19 patients (18 men and 1 woman) in whom treatment was stopped due to irAEs. The median length of follow-up was 16.9 months (range, 4.3-50.2 months). Patient characteristics are shown in Table 1 (Detail information of each patient was showed in Additional file 1: Table S1). Histology showed that 13 patients had squamous cell carcinoma, 4 patients had adenocarcinoma, 1 patient had adenosquamous cell carcinoma, and 1 patient had large-cell carcinoma. All patients had a history of smoking (45 pack-years, range 12.5-116 pack-years) and had a good PS (0 or 1$)$. PD-1/ PD-L1 inhibitors were administered as a second-line treatment for 6 patients, third-line for 8, and as fourthline or later for 5 . Eighteen of 19 patients were treated with nivolumab, one was with atezolizumab. The median number of administration was 7 times (1-70).

\section{Data related to adverse effects}

The AEs were interstitial pneumonitis $(n=10)$, hematological toxicity $(n=2)$, enterocolitis $(n=1)$, diarrhea $(n=1)$, anaphylactic $(n=1)$, hypophysitis $(n=1)$, eruption $(n=1)$, and bleeding $(n=1)$. Fourteen patients (73.7\%) were treated with corticosteroids for irAEs.

\section{Data related to survival}

The confirmed overall response rate during treatment was $21.1 \%$ (4 of 19 patients) (Table 2). One partial response (PR) patient changed to complete response (CR),

Table 1 Patient characteristics

\begin{tabular}{ll}
\hline Characteristics & number of patients $(n=19)$ \\
\hline Gender & Male 18, Female 1 \\
Median age (range), years & 67 (49-80) \\
Histology & Squamous cell carcinoma 13 \\
& Adenocarcinoma 4 \\
& Adenosquamous cell carcinoma 1 \\
& Large cell carcinoma 1 \\
Smoke history & Current 6, Former 13 \\
Pack-years (range) & 45 pack-years(12.5-116) \\
Performance status & PS0 1, PS1 18 \\
Treatment line & 2nd line, 6 \\
& 3rd line, 8 \\
& More than 4th line, 5 \\
PD-1/PD-L1 antibodies & Nivolumab 18 \\
& Atezolizumab 1 \\
Adverse events & Interstitial pneumonia 10 \\
& diarrhea 2 \\
& hematological toxicity 2 \\
& anaphylactic 2, eruption 1, \\
& hemoptysis 1, hypophysitis1 \\
& Steroid 14, others 5 \\
\hline &
\end{tabular}


Table 2 Efficacy of PD-1/PD-L1 inhibitors

\begin{tabular}{|c|c|}
\hline & $n=19$ \\
\hline median number of Cycle (range) & $7(1-70)$ \\
\hline Duration of treatment & 2.8 months ( 1 day-32.9 months) \\
\hline Best response during administration ${ }^{a}$ & PR 4, SD 12, PD 1, NE 2 \\
\hline $\begin{array}{l}\text { Best response including after } \\
\text { discontinuation }{ }^{a}\end{array}$ & $\begin{array}{l}\mathrm{CR} 1, \mathrm{PR} 5, \mathrm{SD} 11, \mathrm{PD} 2 \\
(\mathrm{PR} \rightarrow \mathrm{CR} 1, \mathrm{SD} \rightarrow \mathrm{PR} 2, \mathrm{NE} \rightarrow \\
\mathrm{SD}, \mathrm{PD} 1)\end{array}$ \\
\hline
\end{tabular}

$C R$ complete response, $P R$ partial response, $S D$ stable disease, $P D$ progressive disease, $N E$ not evaluated

${ }^{a}$ According to RECIST 1.1; Confirmed by a later scan performed at least

4 weeks after initial response was observed

and 2 stable disease (SD) patients changed to $2 \mathrm{PR}$ afterwards despite discontinuation of PD-1/PD-L1 inhibitors. The median PFS was 10.2 months (95\% confidence interval $[\mathrm{CI}]=3.2-17.1$ months) and 5.6 months $(95 \%$ $\mathrm{CI}=0-12.2$ months) from the initiation and the discontinuation of PD-1/PD-L1 treatment, respectively (Fig. 1). The median OS was 21.9 months (95\% CI, 9.2-34.6) (Fig. 2). The median PFS according to the confirmed response during administration from treatment was not reached for PR patients and 10.2 months (95\% CI, 3.916.4) for SD patients, respectively $(P=0.04)$ (Fig. 3a). The median PFS after discontinuation was not reached for PR and 4.9 months (95\% CI, 3.7-6.0) for SD patients $(P=0.02)$ (Fig. 3b).

The spider plot showed tumor burden kinetics in patients with NSCLC treated with PD-1/PD-L1 inhibitors $(n=16)$ (Fig. 4). The antitumor effect tended to plateau with 24-week administration of PD-1/PD-L1 inhibitors.
In patients with SD at 24 weeks, a further antitumor effect was not achieved with or without the treatment, except for in 1 patient. Even in those with an antitumor effect, 2 of 4 cases that had stopped the treatment within 8 weeks showed aggravated disease with the appearances of new lesions afterwards. The other 2 cases showed a durable response (8-12 month) with the ultimate appearance of new lesions. The patients with $\mathrm{PR}$ at 12 weeks in whom the administration was continued for 12-24 weeks had good prognoses.

\section{Discussion}

One of the major issues with ICIs is determining the treatment duration has the best balance of high efficacy and low toxicity. The present study evaluated the efficacy of anti-PD-1/PD-L1 antibodies after their discontinuation in patients with NSCLC and estimated the optimum period of treatment, considering risks and benefits. To our knowledge, this is the first study to investigate the duration for which anti-PD-1/PD-L1 antibodies should be continued. Two findings from the present study warrant mention. First, the prognoses in PR patients were completely different from those in SD patients. Second, the PR patients had good prognoses as long as the agents had been administered for a certain period. Our findings suggest that the appropriate period of prescription was 3 to 6 months in patients in whom AEs occurred.

Immunotherapy including anti-PD-1/PD-L1 antibodies has the potential for long-term disease control through the activation of the patients' own immune system against cancer cells in several kinds of cancer [7-13]. The
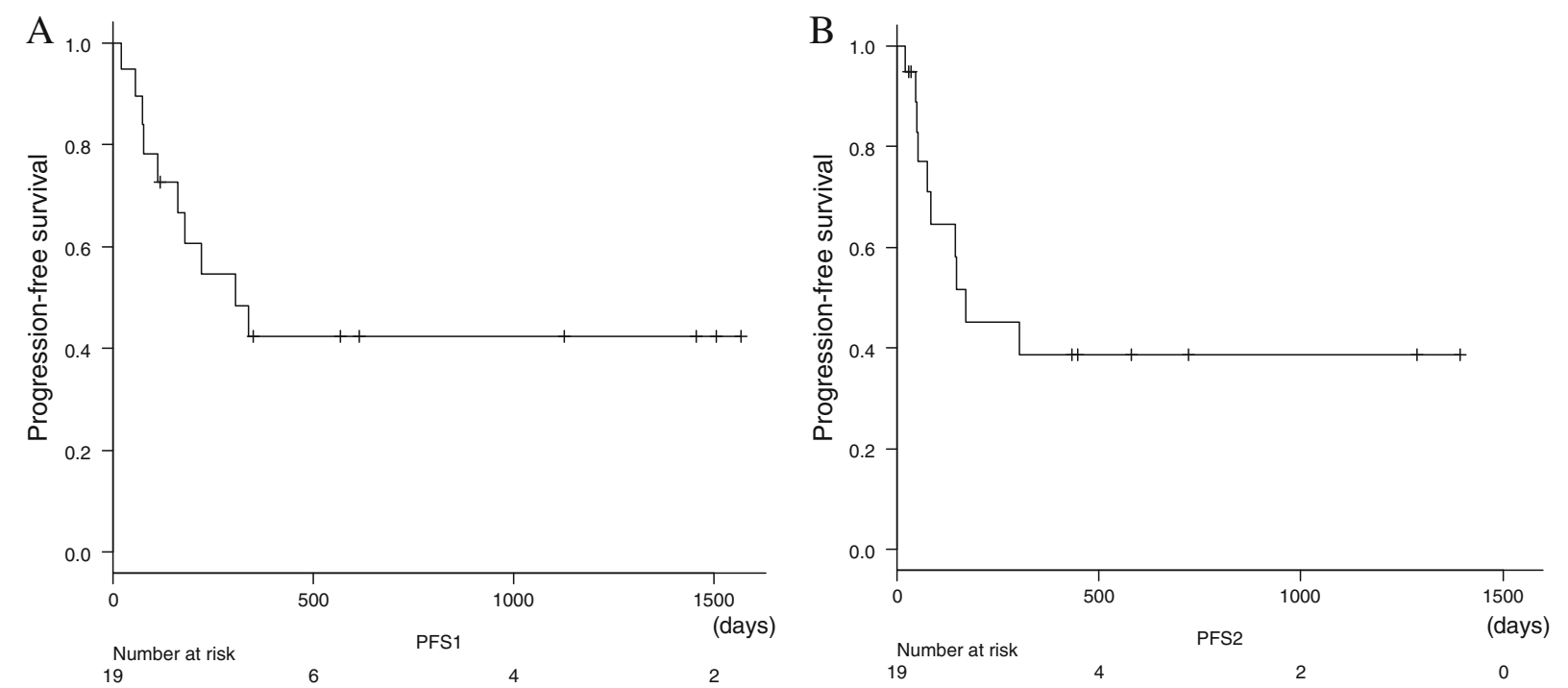

Fig. 1 Kaplan-Meier curves of progression-free survival (PFS). a PFS from the treatment. b PFS from the discontinuation 


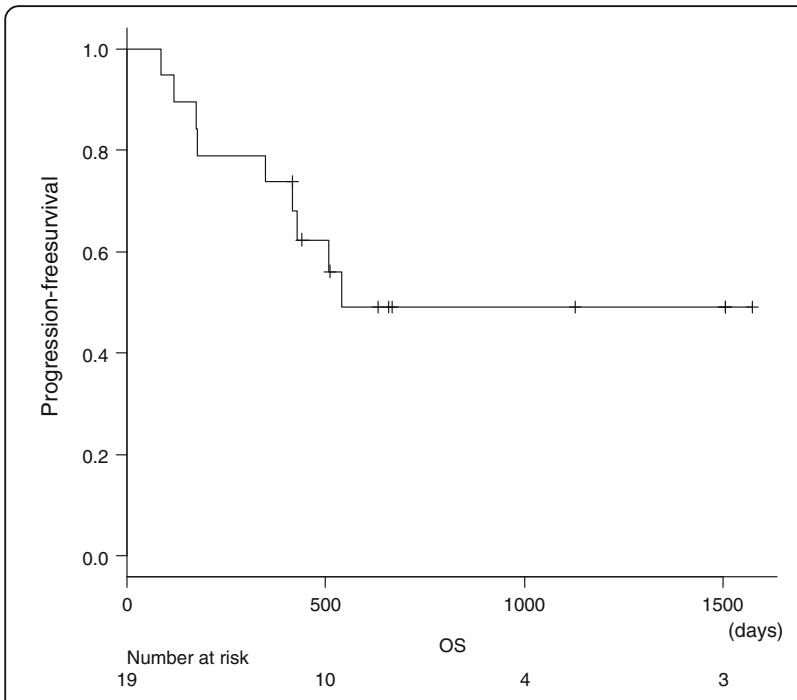

Fig. 2 Kaplan-Meier curves of overall survival (OS)

Kaplan-Meier curves of PFS showed that the slope of the curve flattened out after 6 months for patients treated with PD-1/PD-L1 antibodies [11, 14]. It has also become clear that the antitumor effect lasted even if the ICIs were stopped due to AEs or the prescribed treatment period expired [10-15]. In our study, the antitumor effect tended to fluctuate in the first 8-12 weeks and plateaued with 24-week administration of PD-1/PD-L1 inhibitors. Most of cases with SD at 8-12 weeks didn't show PR, even if antibodies were continued afterward. Furthermore, 1 patient with SD at 24 weeks showed disease progression 20 weeks after stopping treatment. These data suggested that $\mathrm{SD}$ cases ought to be treated for as long as possible.
This may explain why the PFS was completely different between cases with SD and PR.

In contrast, even if there was a good antitumor effect at first, most patients showed aggravation if treatment was stopped within 8 weeks. The patients with PR at 8 12 weeks in whom the administration was continued for 12-24 weeks at least seemed to have a good long-term response. Syukuya et al. reported that the landmark PFS correlated with the OS, with a longer landmark PFS at 24 weeks being the best predictor of the survival in patients with NSCLC treated with anti-PD-1/PD-L1 antibodies [16].

Recently, CheckMate 153 (phase IIIB/IV study) evaluated the clinical benefit of a fixed duration (1 year) of nivolumab treatment vs. continuous treatment in patients with previously treated advanced NSCLC as a secondary endpoint. The result showed that patients with continuous treatment of nivolumab for more than 1 year had a significantly better prognosis than those with fixed-duration treatment $[17,18]$. However, while some cases showed a durable response in the discontinuation group, others showed aggravated disease afterwards despite continuous treatment. These data suggest that the immune response depends on the individual and cannot be stated unconditionally. Research for the acquired resistance mechanisms to ICI is also under way. Optimal use of ICI will hinge on the identification of mechanistic biomarkers of response, irAE, and resistance [19].

ICIs induce various types of irAEs, including late-onset irAEs after long-term prescription [17, 20-24]. Santini et al. reported retreatment with anti-PD-1/L1 therapy resulted in recurrent or new irAEs in half of patients with
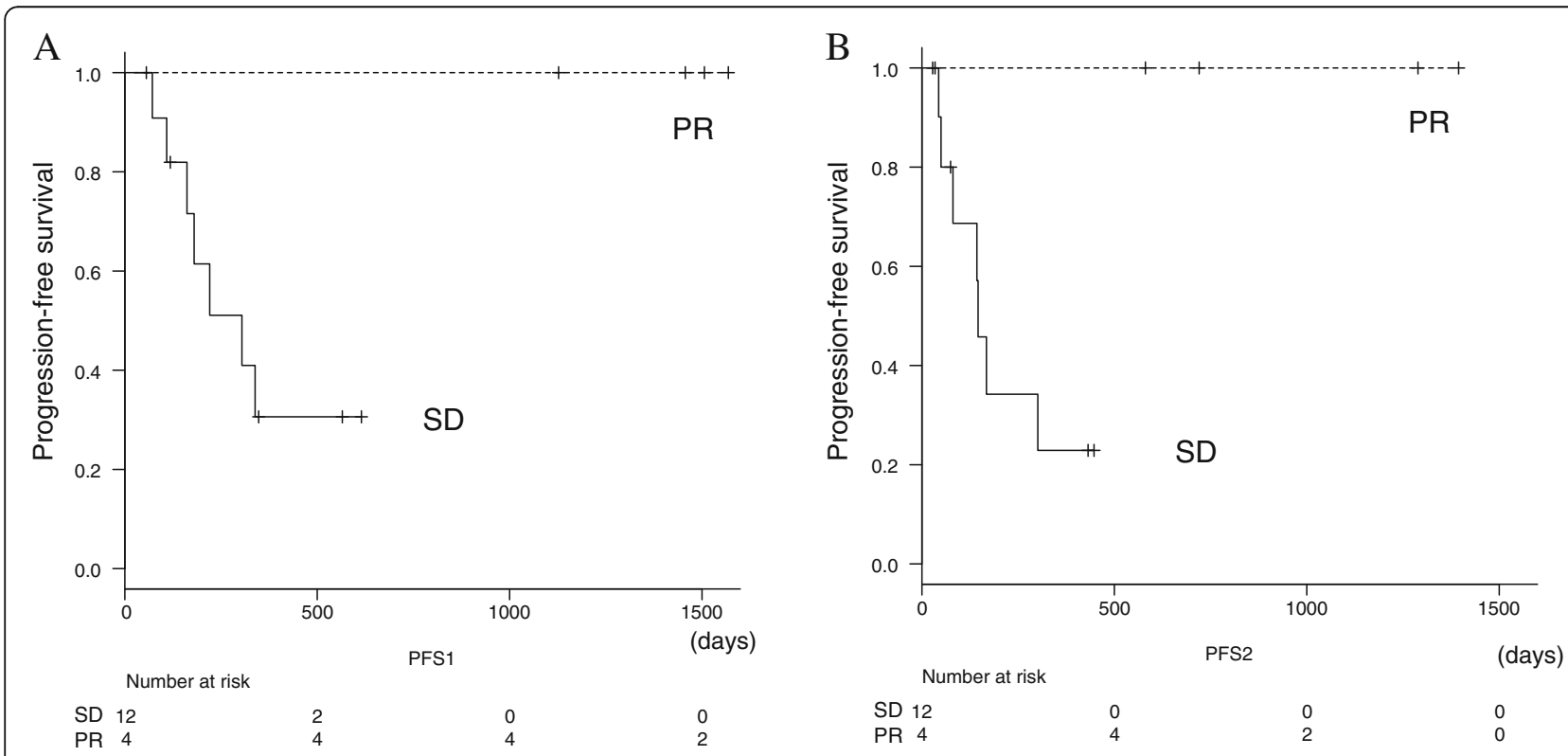

Fig. 3 Kaplan-Meier curves of PFS according to the confirmed response during treatment. a PFS from the treatment. $\mathbf{b}$ PFS from the discontinuation 


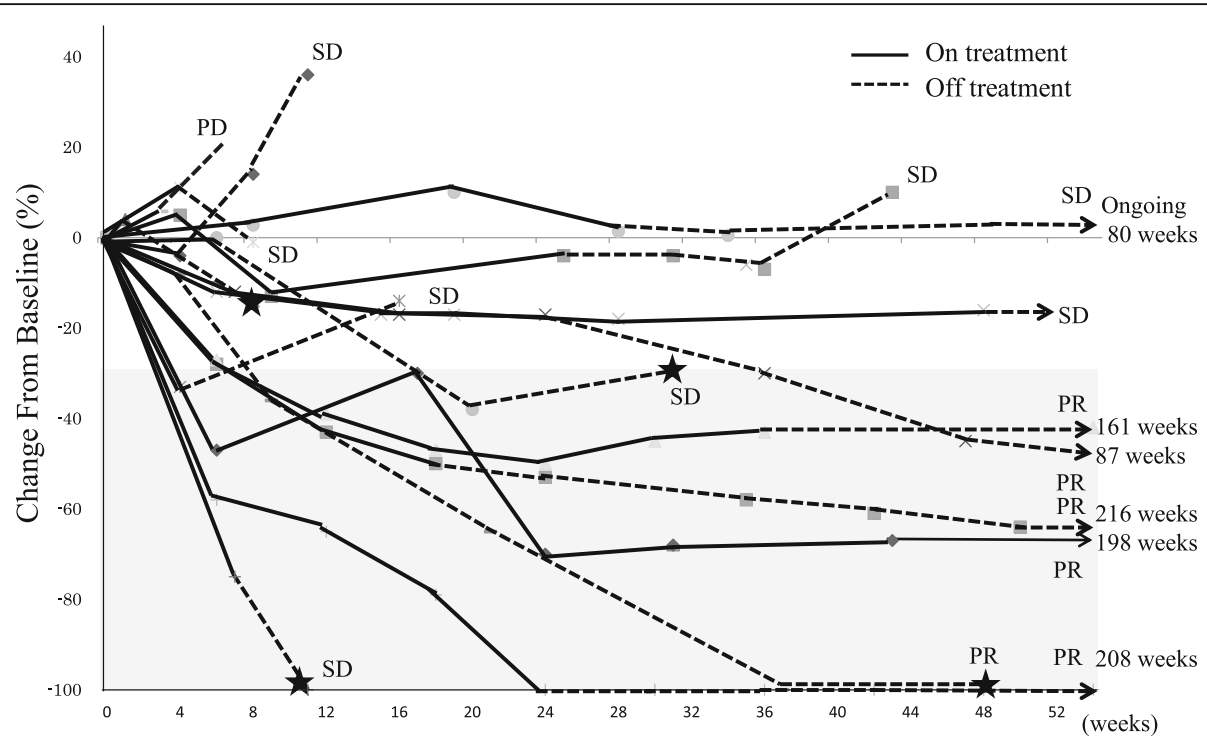

Fig. 4 Spider plot. Tumor burden kinetics in patients with advanced non-small-cell lung cancer treated with PD-1/PD-L1 therapy. Baseline tumor measurements are standardized to zero. Tumor burden was measured as sum of longest diameters of target lesions compared with baseline. Percent change in target lesion tumor burden from baseline over time. Only includes patients with baseline target lesion and one or more post baseline target lesion assessments with no missing value $(n=16)$. Gray zone denote more than $30 \%$ decrease. Solid line and dotted line indicate on treatment and off treatment respectively. Star show occurrence of new lesion

irAEs that had improved. Furthermore, among those with CR/PR prior to onset of irAEs, the PFS and OS were similar in the retreatment and discontinuation cohorts [25]. These present and previous findings suggest that patients who were apparent responders prior to the occurrence of AEs might not need retreatment. Given the risks and benefits, we must decide on whether or not to continue treatment on a case-by-case basis.

The present study has several limitations, such as its retrospective design and small population. Furthermore, we targeted patients who had been unable to avoid stopping treatment due to AEs, with no cases having been stopped at the patient's wish at our institutions. The development of irAEs is reportedly associated with the survival outcome in patients with NSCLC receiving nivolumab treatment [26].

Since we focused on the patients who discontinued ICIs due to AEs, our result might apply only to select patients with a good prognosis. However, despite these limitations, our results may support suggestions to solve a major clinical problem associated with ICIs.

\section{Conclusion}

The PFS of the PR patients was completely different from that of the SD in AE occurred patients. The cases with a disease status better than PR at the time of $\mathrm{AE}$ occurrence tended to show a durable response after the discontinuation of PD-1/PD-L1 inhibitors.

\section{Additional file}

Additional file 1: Characteristics and clinical course of each patient. (XLSX $19 \mathrm{~kb})$

\section{Abbreviations}

AEs: Adverse events; CR: Complete response; CT: Computed tomography; ECOG: Eastern Cooperative Oncology Group; ICls: Immune checkpoint inhibitors; irAEs: immune-related adverse events; NSCLC: Non-small cell lung cancer; OS: Overall survival; PFS: Progression-free survival; PR: Partial response; PS: Performance status; RECIST 1.1: Response Evaluation Criteria in Solid Tumors; SD: Stable disease

\section{Acknowledgements}

The authors would like to express their gratitude to the participating patients and to the members of the HANSHIN Oncology Group.

Funding

No funding was acquired for this study.

\section{Availability of data and materials}

The datasets used and/or analyzed during the current study are available from the corresponding author on reasonable request.

\section{Authors' contributions}

This study was conceived by SN, MT, NK, and SY. MT, TI, MT, YA, TU, YU, YH, and AH collected data. MT performed data analysis and wrote the manuscript. All authors approved the final manuscript.

\section{Ethics approval and consent to participate}

This research conformed to the provisions of the Declaration of Helsinki. This retrospective study was approved by the ethics committee of Kobe University (approval No.160122) and each institutions. Consent to participate: this is a retrospective study and consent to participate was waived by the Ethics Committee of Kobe University and each institution. 


\section{Consent for publication}

Not applicable.

\section{Competing interests}

The authors declare that they have no competing interests.

\section{Publisher's Note}

Springer Nature remains neutral with regard to jurisdictional claims in published maps and institutional affiliations.

\section{Author details}

'Department of Respiratory Medicine, Kobe University Graduate School of Medicine, 7-5-1, Kusunoki-Cho, Chuo-Ku, Kobe-city, Hyogo 650-0017, Japan. ${ }^{2}$ Department of Medical Oncology, Hyogo Cancer Center, 13-70 Kitaoji-cho, Akashi-shi, Hyogo 673-8558, Japan. ${ }^{3}$ Department of Thoracic Oncology, Osaka International Cancer Institute, 3-1-69 Otemae, Chuo-Ku, Osaka-shi, Osaka 541-8567, Japan. ${ }^{4}$ Department of Thoracic Oncology, National Hospital Organization Toneyama National Hospital, 5-1-1 Toneyama, Toyonaka, Osaka 560-8552, Japan. ${ }^{5}$ Department of Thoracic Oncology, Hyogo Cancer Center, 13-70 Kitaoji-cho, Akashi-shi, Hyogo 673-8558, Japan. ${ }^{6}$ Department of Medical Oncology, Kobe City Medical Center General Hospital, 2-1-1 2 Minatojima -Minamimachi, Chuo-ku, Kobe 650-0047, Japan.

Received: 5 April 2018 Accepted: 13 September 2018 Published online: 03 October 2018

\section{References}

1. Francisco LM, Sage PT, Sharpe AH. The PD-1 pathway in tolerance and autoimmunity. Immunol Rev. 2010;236:219-42.

2. Pardoll DM. The blockade of immune checkpoints in cancer immunotherapy. Nat Rev Cancer. 2012;12:252-64.

3. Iwai $Y$, Ishida M, Tanaka Y, Okazaki T, Honjo T, Minato N, et al. Involvement of PD-L1 on tumor cells in the escape from host immune system and tumor immunotherapy by PD-L1 blockade. Proc Natl Acad Sci U S A. 2002;99:12293-7.

4. Dong H, Strome SE, Salomao DR, Tamura H, Hirano F, Flies DB, et al. Tumorassociated B7-H1 promotes T-cell apoptosis: a potential mechanism of immune evasion. Nat Med. 2002;8:793-800.

5. Freeman GJ, Long AJ, Iwai $Y$, Bourque $K$, Chernova T, Nishimura $H$, et al. Engagement of the PD-1 immunoinhibitory receptor by a novel B7 family member leads to negative regulation of lymphocyte activation. J Exp Med. 2000;192:1027-34.

6. Larkin J, Lao CD, Urba WJ, McDermott KDF, Horak C, Jiang J, et al. Efficacy and safety of nivolumab in patients with BRAF V600 mutant and BRAF wildtype advanced melanoma: a pooled analysis of 4 clinical trials. JAMA Oncol. 2015;1:433-40

7. Topalian SL, Sznol M, McDermott DF, Kluger HM, Carvajal RD, Sharfman WH, et al. Survival, durable tumor remission, and long-term safety in patients with advanced melanoma receiving nivolumab. J Clin Oncol. 2014;32:1020-30.

8. Brahmer J, Reckamp KL, Baas P, Crinò L, Eberhardt WE, Poddubskaya E, et al. Nivolumab versus docetaxel in advanced squamous-cell non-small-cell lung cancer. N Engl J Med. 2015;373:123-35.

9. Borghaei H, Paz-Ares L, Horn L, Spigel DR, Steins M, Ready NE, et al. Nivolumab versus docetaxel in advanced nonsquamous non-small-cell lung cancer. N Engl J Med. 2015;373:1627-39.

10. Garon EB, Rizvi NA, Hui R, Leighl N, Balmanoukian AS, Eder JP, et al. Pembrolizumab for the treatment of non-small-cell lung cancer. N Engl J Med. 2015;372:2018-28.

11. Gettinger SN, Horn L, Gandhi L, Spigel DR, Antonia SJ, Rizviet NA, et al. Overall survival and long-term safety of nivolumab (anti-programmed death 1 antibody, BMS-936558,ONO-4538) in patients with previously treated advanced non-small-cell lung cancer. J Clin Oncol. 2015;33:2004-12.

12. Herbst RS, Baas P, Kim DW, Felip E, Pérez-Gracia JL, Han JY, et al. Pembrolizumab versus docetaxel for previously treated, PD-L1-positive, advanced non-small-cell lung cancer. (KEYNOTE-010): a randomised controlled trial. Lancet. 2016;387:1540-50.

13. Gettinger S, Horn L, Jackman D, Spigel D, Antonia S, Hellmann M, et al. Fiveyear follow-up of Nivolumab in previously treated advanced non-small-cell lung Cancer: results from the CA209-003 study. J Clin Oncol. 2018;36:1675-84.

14. Gettinger S, Rizvi NA, Chow LQ, Borghaei H, Brahmer J, Ready N, et al. Nivolumab monotherapy for first-line treatment of advanced non-small-cell lung Cancer. J Clin Oncol. 2016;34:2980-7.
15. Robert C, Long GV, Brady B, Dutriaux D, Maio M, Mortier L, et al. Nivolumab in previously untreated melanoma without BRAF mutation. N Engl J Med. 2015;372:320-30.

16. Shukuya T, Mori K, Amann JM, Bertino EM, Bertino EM, Yagishita S, Kanemaru R, et al. Relationship between overall survival and response or progression-free survival in advanced non-small cell lung Cancer patients treated with anti-PD-1/PD-L1 antibodies. J Thorac Oncol. 2016;11:1927-39.

17. Sun X, Roudi R, Chen S, Fan B, Li HJ, Zhou M, et al. Immune-related adverse events associated with PD-1 and PD-L1 inhibitors for nonsmall cell lung cancer: protocol for a systematic review and meta-analysis. Medicine. 2017;96:e8407.

18. Spigel DR, McLeod M, Hussein MA, Waterhouse DM, Einhorn L, Horn L. et al. 12970 Randomized results of fixed-duration (1-yr) vs continuous nivolumab in patients (pts) with advanced non-small cell lung cancer (NSCLC). Ann Oncol. 2017;28(suppl_5). https://doi.org/10.1093/annonc/mdx380.002. Published: 18 September 2017.

19. Lam WS, Wang LZ, Roudi R, Yong WP, Syn NL, Sundar R. Resisting resistance to cancer immunotherapy. Thorac Cancer. 2018;9:507-8.

20. Boutros C, Tarhini A, Routier E, Lambotte O, Ladurie FL, Carbonnel F, et al. Safety profiles of anti-CTLA-4 and anti-PD-1 antibodies alone and in combination. Nat Rev Clin Oncol. 2016;13:473-86.

21. Champiat S, Lambotte O, Barreau E, Belkhir R, Berdelou A, Carbonnel F, et al Management of immune checkpoint blockade dysimmune toxicities: a collaborative position paper. Ann Oncol. 2016;27:559-74.

22. Nishino M, Ramaiya NH, Awad MM, Sholl LM, Maattala JA, Taibi M, et al. PD-1 inhibitor-related pneumonitis in advanced cancer patients: radiographic patterns and clinical course. Clin Cancer Res. 2016:22:6051-60.

23. Naidoo J, Wang X, Woo KM, lyriboz T, Halpenny D, Cunningham J, et al. Pneumonitis in patients treated with anti-programmed death-1/ programmed death ligand 1 therapy. J Clin Oncol. 2017;35:709-17.

24. Barlesi F, Steins M, Horn L, Ready N, Felip E, Borghaei $H$, et al. Long-term outcomes with nivolumab vs docetaxel in patients with advanced NSCLC:Checkmate 017 and Checkmate 057 2-y update. Ann Oncol. 2018;27(suppl 6):1215PD.

25. Santini FC, Rizvi H, Wilkins O, Voorthuysen MV, Panora E, Halpennyet D, et al. Safety of retreatment with immunotherapy after immune-related toxicity in patients with lung cancers treated with anti-PD-(L)1 therapy. J Clin Oncol. 2017;35(suppl; abstr 9012).

26. Haratani K, Hayashi H, Chiba Y, Kudo K, Yonesaka L, Kato R, et al. Association of Immune-Related Adverse Events with Nivolumab Efficacy in non-small-cell lung Cancer. JAMA Oncol. 2018;4:374-8.

\section{Ready to submit your research? Choose BMC and benefit from:}

- fast, convenient online submission

- thorough peer review by experienced researchers in your field

- rapid publication on acceptance

- support for research data, including large and complex data types

- gold Open Access which fosters wider collaboration and increased citations

- maximum visibility for your research: over $100 \mathrm{M}$ website views per year

At BMC, research is always in progress.

Learn more biomedcentral.com/submissions 